\title{
Mob: A Scripting Language for Mobile Agents Based on a Process Calculus
}

\author{
Hervé Paulino ${ }^{1}$, Luís Lopes ${ }^{2}$, and Fernando Silva ${ }^{2}$ \\ 1 Department of Informatics. Faculty of Sciences and Technology. \\ New University of Lisbon. 2825-114 Monte de Caparica, Portugal. \\ herve@di.fct.unl.pt \\ 2 Department of Computer Science. University of Oporto. \\ Rua do Campo Alegre, 823, 4150-180 Porto, Portugal.
}

\{lblopes, fds\}@ncc.up.pt

\begin{abstract}
Mobile agents are the latest software technology to program flexible and efficient distributed applications. Most current systems implement semantics that are hard if not impossible to prove correct. In this paper we present Мов, a scripting language for Internet agents encoded on top of a process calculus and with provably sound semantics.
\end{abstract}

\section{Introduction}

This paper presents a scripting language, MoB, for programming mobile agents in distributed environments. The semantics of the language is based on the DiTyCO (Distributed TYped Concurrent Objects) process calculus [1], whose implementation provides the run-time for the language. In particular, we rely on it for interprocess communication and code migration over the network.

The development of mobile agents requires a software infrastructure that provides migration and communication facilities, among others. Current frameworks that support mobile agents are mostly implemented by defining a set of Java classes that must then be extended to implement a given agent behavior, such as Aglets [2], Mole [3], or Klava [4]. Мов, on the other hand, is a simple scripting language that allows the definition of mobile agents and their interaction, an approach similar to D'Agents [5]. However, Мов applications may interact with external services programmed in many languages such as Java, C, TCL or Perl. The philosophy is similar to that used in the MIME type recognition. The runtime engine matches a file type against a set of internally known types and either launches the correspondent application or simply executes the code.

Mов also differs from other mobile agent frameworks in the fact that it is compiled into a process-calculus core language, and its semantics can be formally proved correct relative to the base calculus. Thus, the semantics of Mов programs can be understood precisely in the context of concurrency theory. 


\section{Language Description}

In this section we describe the basic language abstractions.

\subsection{Agents}

The MoB programming language is a simple, easy-to-use, scripting language. Its main abstractions are mobile agents that can be grouped in communicators allowing group communication and synchronization. Programming an agent involves implementing two steps: the init and the do methods. Method init consists of a setup that will execute before the agent starts its journey. It is usually used to assign initial values to the agent's attributes. The do method defines the agent's actions/behavior on the journey.

Agents have several built-in attributes: owner carrying the identification of the agent's owner; itinerary carrying the agent's travel plan; and strategy carrying the definition of a strategy of how the itinerary must be traveled. The programmer may define as many new attributes as he wishes. Their usefulness is to hold state to be retrieved when the agent migrates back home. The following example presents the skeleton of a MOB agent definition, Airline. Beside the built-in attributes, a new one, named price, has been defined.

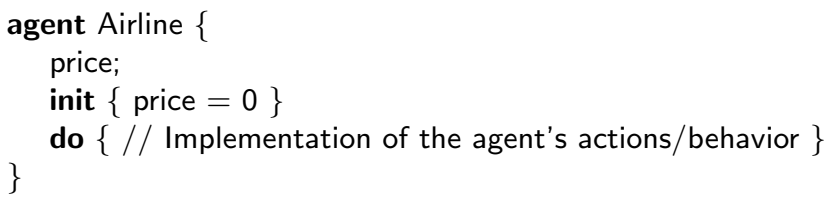

Once the template for an agent is defined an undetermined number of agents can be created. The following example creates an agent named airline owned by johndoe and with home hosts host1 and host2. One can also launch several agents at once using the $\mathbf{- n}$ flag. airlineList will contain the list of the agent's identifiers. Notice that the attribute initialization supplied in the agent constructor will not override the ones in the init section.

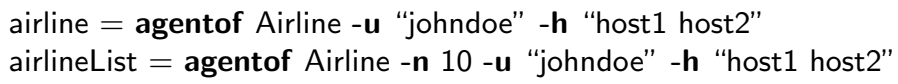

Each agent must be associated to an owner, defined in an entry of the Unix-like file named passwd. An entry for each user contains login, name, password and group membership information. Following the Unix policy for user management, users may belong to groups defined in the groups file, sharing their access permissions. Each Mов enabled host must own both files in order to authenticate each incoming agent. As featured in FTP servers, an agent can present itself as anonymous for limited access to local resources.

\subsection{Communicators}

Communicators are conceptually equivalent to MPI communicators [6] and allow group communication and synchronization. The communicator construct requires the list of agents (eventually empty) that will start the communicator. Other agents may join later. 


\subsection{Instructions}

Most of the statements included in MOB are common in all scripting languages (for, while, if, foreach and switch), the only difference lies in the try instruction, a little different from the usual error catching instructions found in, for instance, TCL. Its syntax is similar to the try/catch exception handler instruction of Java, allowing specific handling of different types of run-time system exceptions. MoB provides instructions to define a mobile agent's behavior and its interaction with other agents and external services. These commands can be grouped in the following main sets:

1. agent state (email, owner, home, itinerary, strategy, hostname, etc) and creation (clone).

2. mobility: go.

3. checkpointing: savestate and getstate.

4. inter-agent communication: asynchronous (send, recv, recvfrom), synchronous (bsend, brecv, brecvfrom), communicator-wide (csend) and multicast (msend). There are variants of these functions for use with the HTTP and SMTP protocols (e.g., httpcsend; smtprecv). These variants are useful to bypass firewalls that only allow connections to ports of regular services.

5. managing communicators: cjoin and cleave.

6. execution of external commands: exec. This functionality allows the execution of commands external to the Mов language. The MоB system features a set of service providers that enable communication through known protocols, such as HTTP, SMTP, SQL and FTP. The interaction with these providers is possible through exec's protocol flag.

MoB's input/output instructions are implemented as a syntactic sugar for the exec instruction. open filename could also be written as exec -p fs open filename. МоB features all the common file input/output commands.

\section{$3 \quad$ Programming Example}

The example defines a network administration agent that performs a virus check by perpetually moving through the hosts. If a virus is found and the installed version of the anti-virus cannot solve the problem, the agent updates the antivirus software and retries to remove the virus. If the problem remains an email is sent to the administrator. This implementation considers that all the anti-virus software is present, thus not protecting the exec calls with try instructions.

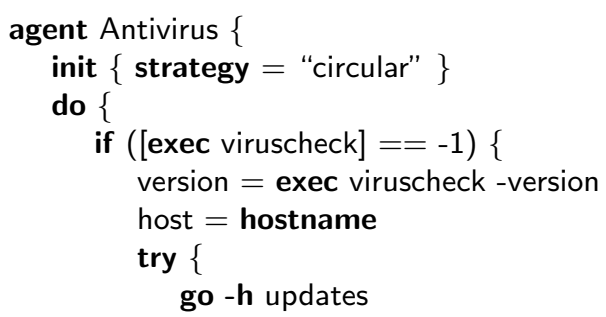




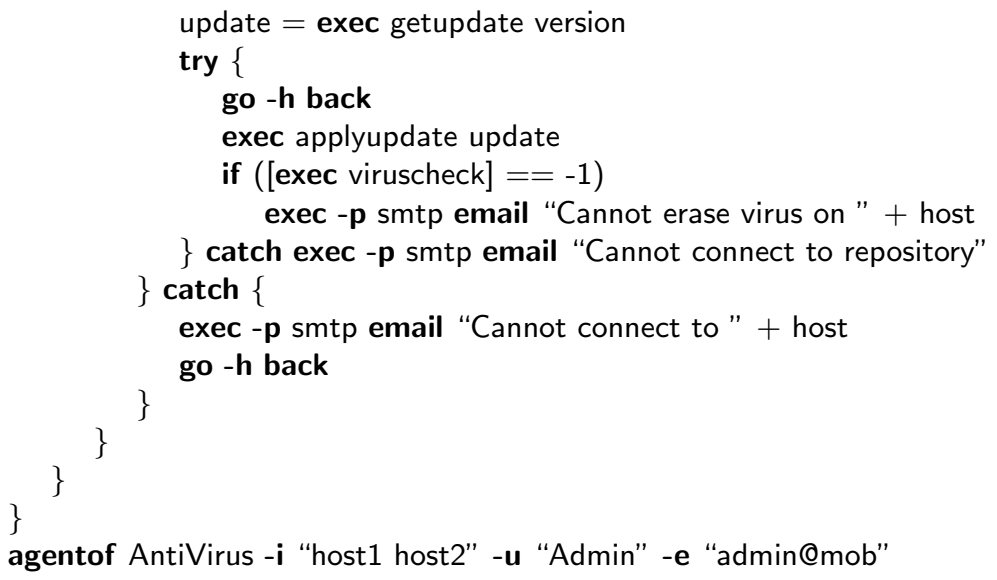

When the agent reaches the end of the do code it migrates to the next host resulting of the application of the strategy over the itinerary. In this case, a circular strategy that moves accross all nodes of the itinerary forever. Although MoB features several strategies, it allows the programming of new ones. An agent's itinerary is seen as an object that can be managed through an iterator. The methods fand previous of the iterator define the MOB traveling strategy.

\section{Future Work}

Mов is currently under implementation. Future work will focus on features such as the implementation of a local exception handling mechanism and the development of external services, such as, recognition/execution of programs in several high-level languages (e.g. Java, C, TCL).

Acknowledgments. This work is partially supported by FCT's project MIMO (contracts POSI/CHS/39789/2001).

\section{References}

1. V. Vasconcelos, L. Lopes, F. Silva: Distribution and Mobility with Lexical Electronic Notes in Theoretical Computer Science 16(3), Elsevier Science Publishers, 1998.

2. Lange D.: Java Aglet Application Programming Interface. IBM Tokyo Research Laboratory. 1997.

3. Straber K., Baumann J., Hohl F.: Mole - A Java Based Mobile Agent System. Inst. for Parallel and Distributed Computer Systems, University of Stuttgart. 1997.

4. Bettini L., De Nicola R., Pugliese R.: Klava: a Java Framework for Distributed and Mobile Applications. Software - Practice and Experience, 32(14):1365-1394, John Wiley \& Sons, 2002.

5. Gray R.: Agent TCL: A flexible and secure mobile-agent system. Department of Computer Science. Dartmouth College. 1996.

6. MPI Forum: The MPI Message Passing Interface Standard. www-unix.mcs.anl.gov/mpi/. 1994 